\title{
Healing Power of Sound
}

\author{
Nils de Mol van Otterloo ${ }^{1}$, Nimit Sudan ${ }^{2}$, Matthew Lyons ${ }^{3}$, Murali Nair ${ }^{4}$ \\ ${ }^{1}$ Fulbright Scholar, Institute of Medical Sciences, Amrita University, \\ Ponekkara, Kochi, Kerala 682041, India \\ ${ }^{2}$ Medical Group, City of Hope, \\ 44105 15th St West, Lancaster, CA 93534, USA \\ ${ }^{3}$ School of Public Health, Georgia State University \\ One Park Place, Atlanta, GA 30303, USA \\ ${ }^{4}$ School of Social Work, University of Southern California, \\ 669 W. 34th Street, Los Angeles, CA 90089, USA
}

\begin{abstract}
This article discusses the healing powers of sound and its diverse applications, ranging from ancient teachings and practices to present-day evidence-based studies. Various cultural, geographical, scientific, spiritual, and religious perspectives have been gathered to illustrate the many ways sound is utilized to improve human wellbeing.
\end{abstract}

Keywords: sound, human well-being, traditional approach, evidence-based.

\section{Introduction}

In comparison with the 400 years of modern academia, the history of homo sapiens spans millennia. Although the academy has excelled in advancing knowledge through the scientific method, it has, either by nature or design, left behind some insights gleaned through traditional modes of understanding left by more ancient and non-Western peoples. In many ways, human knowledge has undergone tremendous positive developments in the last century that eclipse all centuries before it. The achievements of modern medicine, computer science, and aerospace engineering (to name only a few) are not to be diminished. However, traditions that are external to the world of empirical science can still prove to be sources of fruitful insights, and glimpses of this knowledge are beginning to peer through the cracks of the hermetically sealed chamber of academe.

Due to globalization and the concomitant increase in the permeability of national and cultural borders, there are subjects that were previously outside the ken of Western thinking that have become available in popular culture. When the Beatles went to India, they opened many Western minds to the musical, spiritual, and healing world of the Devi. Fifty years later, music therapy is a burgeoning academic field, and there is a growing need for further research into its efficacy and possible applications. When the subject of music therapy and neurological healing is a topic of Keeping
Up with the Kardashians," the time for greater research in arts and the social sciences is now [1]. A good place to begin the understanding of music therapy is by considering the place of disparate cultures in the training of therapists [2]. This is true in music therapy as it is in social work.

It seems, out of all five of the human's anatomical senses, our sense of sound is the strongest. Even in our sleep, when we do not speak, taste, see, or feel, sounds still find their way into our dreams. Our ears are never closed. Some say that music is the universal language of the world, because sounds can deliver powerful messages without the need for symbolic meaning. The global popularity of certain musical artists can attest to this fact. The immense popularity of the Rolling Stones in South America or the increasing interest in Tuvan throat singing among certain American artists are prime examples. Happiness, sadness, anger, regret, reflection, and even peace and violence can all be communicated through sounds.

Different people perceive sounds differently, however, and even nonmusical sounds have a way of resonating with humans and other animals alike. The nighttime sounds of crickets and cicadas dwelling in tall pine trees can be like a sweet lullaby to one person, whereas to another, the lively sounds of a busy, metropolitan expressway can have the same soothing effect. Our perception of sound is key in determining its effect on 
us. Once a sound hits us, it can influence our mood, affect, spirit, level of energy, and even our health. Although some ancient methods of developing and maintaining a healthy state and sense of well-being have become archaic, methods that indirectly or actively incorporate sound are occurring in all inhabited places on Earth and during every time period.

In the modern context, with the growing prevalence of certain kinds of psychiatric disorders, the importance of traditional sound healing methods is ever increasing. Social workers, psychologists, and other social scientists can gain a great deal of understanding about their clients and their craft by exploring the importance of music in culture. Doctors and medical scientists can also benefit a great deal from this research. There are many patients who are permanently affected by neurological disorders that cannot be cured through medications or surgeries. The incidence rates of dementia [3], traumatic brain injury [4], and posttraumatic stress disorder are growing [5]. Music interventions provide a cheap, noninvasive method of treatment for these disorders. Developing best practices for treatment of clients that include music therapies is a promising avenue of research. Finally, how we consider the future of music-based therapies will depend on researchers and clinicians that are prepared to take on the challenge of developing this form of pedagogy [6].

\section{Multicultural Approaches to Sound Healing}

\subsection{A note on scholarship, secularity, and authority}

In the following passages, various disparate metaphysical interpretations of the nature of sound and its impact on human well-being are addressed. It is neither the intention nor the place of our present study to arbitrate the truth value of various propositions regarding the use of sound in multiple cultural contexts, particularly those that are held to be sacred among their practitioners. Rather, it is our intent to present as clearly and faithfully as possible at least one generative interpretation of the use of sound for the purpose of healing from each of the cultural practices that we address. These presentations will be necessarily incomplete, as each culture that we seek to address is in fact a much more complex amalgamation of distinct phenomena than we have the space or scholarly capacity to address. No single historical figure or modern interpreter has absolute authority over any given tradition, and the legacy of these cultural practices is inevitably contested, from both within and outside the community in question. For the purposes of this essay, we will bracket questions of traditional authority or veracity in the context of Western empirical epistemology, instead choosing to present the material selected using the vocabulary in our source material. To that end, source material was selected not based on a judgment of its relative authority, but rather its clarity and applicability to the present undertaking. It is our hope that the examples provided, although representing only a sliver of much larger historical phenomena, will serve to illustrate how sound has been understood as a healing power.

Historical findings regarding the first sound healing methods are incomplete, but cultures on every continent have used music and other sounds in the treatment of physical, mental, and emotional challenges and in spiritual practice. One of the earliest documented uses of sound is South American whistling vases, or Chimu, that have been found in archaeological sites from $1000 \mathrm{BC}$ until the time of the conquistadors in Colombia, Peru, and other sites [7]. The use of traditional sound healing in Native American cultures, Hinduism, Buddhism, and other contexts shapes the modern-day methods of sound healing, some of which that have proven effective in every setting from ceremonial procedures to modern hospitals. To fully comprehend the relevance of sound and music healing, it will be helpful at this juncture to discuss some examples of early cultures that have used it around the world.

\subsection{Native American traditions}

The United States and Canada are home to two and a half million indigenous people who make up more than 500 different nations [8]. Among these rich and varied cultures, there is no single shared religion or set of spiritual beliefs; there is a diverse array of oral traditions that are passed through generations [8]. Medical care, such as sound healing, involves a connection with nature and the universe [8]. The methods of sound healing used for a specific patient depend on how that patient's oral tradition and spiritual belief is expressed.

Some sound healing in Native American traditions involve ceremonial procedures. For example, if a Native American person is hospitalized, on occasion, a 
singer will visit the hospital with a hand drum to sing a prayer song [8]. Due to the volume of the music, a special room is generally provided by the hospital to properly perform the ritual, unless there is an agreement between the singer and the doctors to have a quiet ceremony [9]. The drum represents the heartbeat of Mother Earth. Tribal singers are considered very emotionally healing for the patient. A patient may even bring audio with a prayer song to listen to while being cared for [8]. Hearing seems to the last remaining sense when a patient is in a coma or approaching death. Therefore, drums are played and rattles are shaken as reassurance so the patient knows that she is not alone or forgotten [8]. Rattles are also used to dispel bad spirits, bring forth good spirits, and help return missing pieces of one's soul [9].

One of the most popular items derived from Native American cultures and traditions is the Native American flute. The flute, a wooden musical instrument developed by indigenous people in the Americas, was actively involved in the treatment of the sick [10]. The sounds of the flute have reportedly been used in present-day hospice care and even in the treatment of trauma-related symptoms [11]. Even for the musician, the Native American flute reportedly displays healing properties. It has been observed that playing the flute can help lung functioning, posture, breathing coordination, and even respiratory parameters such as asthma in children [11]. The Native American flute is still heard today in places such as meditation centers, museum shops, and yoga studios [11].

Native American sound focuses closely on influences from nature and natural sounds. Marjorie de Muynck, a multi-instrumental musician and music composer, wrote two textbooks on the subject of sound healing [12]. Muynck uses various instruments and slowed down vibrations of plant and animal sounds to make music. Muynck calls this music vibrational healing music and has stated that it "gives us an opportunity to re-connect with elemental voices and re-attune to the slower pace and harmonious rhythms of the natural world" [12].

\subsection{Hinduism}

Hinduism is a religion, or rather a complex and diverse amalgamation of religious traditions, practiced by nearly a billion people [13]. The majority of people of Hindu faith inhabit India, but there are large populations in United States, Great Britain, Canada, the Caribbean, Australia, and East Africa that also practice Hinduism [13]. Practitioners of Hinduism rely heavily on the use of short chants called mantras. The word "mantra" is a derivative of Sanskrit and translates as "mind device" [14]. The Hindu scriptures defines mantra as "that which, when contemplated and repeated, will offer protection" [14]. Like Native American traditions, Hindus use sounds for healing, but Hindus specifically incorporate the use of mantras into protective ceremonies and for overall health and wellbeing. Sanskrit mantras use the human voice to influence what practitioners regard to be channels of spiritual energy processing systems known as chakras [14]. Mantras can be done with any number of people, in any quiet place where meditation is possible [14]. Mantras can be recited in two ways to yield significant results: 108 times in a row twice a day for 40 days or 125,000 times at any pace [14]. "Then they can be repeated regularly as needed, even off and on during the day, to help us maintain calm and focus of the mind, or to direct us towards the particular goal that we are seeking through repeating the mantra" [15]. The sacred "Om" sound is the most holy of all the Hindu mantras. This sound is used to signify the "ascending power of pure consciousness" [15]. The popular symbol for Om also depicts the trinity of God in Brahma (the creator), Vishnu (the preserver), and Shiva (the destroyer) [13].

Mantras are used in yoga as the most common and powerful meditation method [15]. Seed sounds are short, powerful mantras, each associated with an anthropomorphized deity such as Shiva, Saraswati, or Lakshmi [14]. Some of the primary seed sounds are "shrim," "eim," "klim," “dum," "kreem," and "gum" [14]. Shakti bija mantras are thought to hold forces of nature such as the energy of the sun, the moon, electricity, and magnetism, and as inner potentials of divine light, love, and wisdom [15]. Shakti bija mantras hold the power of the Kundalini force, considered to be the higher evolutionary power of consciousness among Hindu practitioners [15]. Other examples of Shakti mantras are "aim," which calls on energy of sound for creative power of knowledge and guidance; "hrim," which calls on solar energy for illumination and reflection; and "hum," which calls on the power of fire to purify, protect, and remove negativity [15]. A Shakti mantra is used during meditation so that it may draw the mind into deep awareness [15]. The mantra is 


\section{International Journal of Innovative Research in Medical Sciences (IJIRMS) \\ ISSN (Online): 2455-8737, Vol. 01, Issue 06, August 2016 \\ Available online at $-\underline{w w w . i j i r m s . i n}$}

repeated many times before meditation to put the mind in a meditative state [15]. The individual then keeps mantra repeating in his subconscious while the meditation proceeds, to help withstand the process [15]. Several Shakti bija mantras can also be used together as meditation mantras, often in groups of three to seven bijas.

Mantras are not a replacement for other medical care, but they serve to enhance life components that are already available [14]. Specific healing mantras are used for enhancement in certain life situations. These mantras are used for certain purposes such as general help, attracting relationships, attracting abundance and good luck, healing, invoking feminine power, and spiritual growth [14].

In addition to Hindu sacred healing practices, India also plays host to musical healing in the form of its classical music. In Indian classical music, ragas are specific forms of tonic intervals that are capable of causing the listener to have an emotional response [16]. A 2015 study at the University of Reading in Reading, United Kingdom, investigated the association of tonic intervals, tempo, and rhythmic regularity in ragas with emotional response [16]. Interestingly enough, the results showed that all ragas created a calming effect and anger remained the lowest-rated emotion category [16]. The authors stated: "This is in consensus with research on Western music which shows that negative emotions like anger which are regularly experienced in everyday life are only rarely experienced in response to music" [16].

\subsection{Buddhism}

Buddhism has existed for more than 2,500 years [17]. Buddhist teachings, sacred writings, theories, and practice have been incorporated in many cultures throughout Asia [17]. Although it represents a broad and diverse set of cultural phenomena, it can be helpful to think of Buddhism as categorized into four types: Southeast Asian Theravada Buddhism, East Asian Mahayana Buddhism, and Indo-Tibetan Mahayana and Vajrayana Buddhism [17]. Wallace [18] stated that the goal of Buddhist practice is "the realization of a state of well-being that is not contingent on the presence of pleasurable stimuli, either external or internal." Although any religious tradition as diverse as Buddhism cannot be easily rendered according to a single teleology, Wallace's representation may serve as a generative starting point. As the Dalai Lama commented, "I believe that the very purpose of our life is to seek happiness. Whether one believes in religion or not, whether one believes in this religion or that religion, we all are seeking something better in life. So, I think, the very motion of our life is towards happiness" [19].

The Buddhist text Mahaprajnaparamita Sutra states: "In order to build a Pureland, the Bodhisattvas make use of beautiful music to soften people's heart, as with their heart softened, people's mind are more receptive and thus easier to educate and transform through the teachings" [20]. Some Buddhists believe that music makes one more prepared to become enlightened and at peace. Some worship music so much, in fact, that they use music as one of the offerings to Buddha [20]. Buddhist music commonly uses a relaxed and easy pace, soft tones, and a dignified, solemn manner [20]. According to the Vinaya in Ten Recitations, regularly listening to Buddhist music can give the following five benefits: (a) a reduction in bodily fatigue, (b) less confusion and forgetfulness, (c) a reduction in mental weariness, (d) a more elegant voice, and (e) greater ease in both personal expression and communication [20]. A Record of the Buddhist Religions as Practiced in India and the Malay Archipelago explains six merits that can be obtained by singing and chanting on a regular basis: (a) knowledge of the depth and extent of the Buddha's virtue, (b) an intuitive realization of the truths of the dharma, (c) a reduction in negative or harmful habits of speech, (d) a clearer and healthier respiratory system, and (e) a mind freer from fear and anxiety, and (f) longevity and improved health [20].

As previously stated, some cultures use traditional sound healing to increase the emotional response of an individual. Buddhist music is not necessarily used in that way. Rather music is often used "to conserve emotional energy, calm thoughts, reduce desire, and allow practitioners to see their true nature with a clear mind" [20]. Certain Indian religious traditions teach that God first created sound, and all other things in the world came from these sound frequencies [20]. All existence derives from these sounds, which give rise to mantras when there is a desire to communicate [20]. Matter is said to have been created from sound and the aforementioned Om, the most sacred of all sounds [20]. Buddhist Indian traditions teach that Om preceded the universe and created the gods [20]. 
Pirit, paritta, or paritrāna is the protective doctrines preached by the Buddha that include ceremonial chants [21]. It involves intoned recitation from Buddhist scriptures in the Pali language [21]. Deriving from the suffering of the people, plants, and animals during the reigning of King Upatissa in $410 \mathrm{AD}$, pirits were used to rid communities of natural and supernatural dangers [21]. The aim of pirits is to protect, heal, and bless a person or community [21]. Dona stated, "A pirit chant is considered appropriate before the commencement of any new undertaking or in times of fear, an epidemic, a sickness, a long journey, the launching of a new business, before marriage, or birth giving" [21]. Traditionally, Buddhist monks will be invited to a temple, where they will visit a special pavilion decorated with white or silver paper and coconut leaves [21]. The color white is emphasized, which they believe symbolizes purity, holiness, and prosperity [21]. There, they will take turns chanting pirit in a low pitch for individuals or communities [21]. These ceremonies can last anywhere from 1 hour to 7 days depending on the extent of the problem they are facing [21].

Pirit chanting is done in a nonrhythmic manner. It is dialogue-like in form, so to hear it as music is subjective and determined by the listener's perception [21].

Monks gather in two groups and compete in increasing tempo and pitch so that one group sounds more aggressive than the other [21]. Buddhists believe that the monk's vocal cord vibration chases away the devil's influences; harmonizes one's mind, body, and soul; and removes fear and doubt [21]. Pregnant women often ask for a monk to chant a pirit before delivery [21].

The Six Healing Sounds Qigong, also known as the Six Syllable Secret, is a Buddhist technique developed in the 6th century that uses breathing and sound in a way that is believed by practitioners to rid the body's organs of toxins and stagnant qi. As for the procedure, a person sits on the edge of a hard chair or lies on her back. The person then meditates and breathes for a few minutes. The person then focuses her mind on an organ. She tries to feel the location of the organ in her body. If a person is unsure of the location of an organ, she may use an anatomical chart to assist her. The person then breathes in fresh qi through the nose, and imagines the qi healing her body. She breathes stagnant qi out through the mouth, while chanting a particular syllable depending on which organ is being focused on. She should try to feel the specific organ vibrate in harmony with the sounding of the syllable. The breathing is repeated three to six times for each organ before moving on to the next one. If a specific organ is still in pain, the breathing exercise may be repeated for that particular organ. The organs and their corresponding sounds to remove stagnant qi are as follows: for the lungs, "see-ahh"; for the kidneys, "chrroooeee"; for the liver, "shuuu" (the mouth should form a U shape); for the heart, "he" (as in her without the r); for the spleen, "hooo" (like the word "who"); and for the entire torso and head, "seee."

\subsection{Traditional music healing}

In the previous section, we attempted to provide a general overview of a few musical healing traditions that may be of interest to researchers and practitioners in modern music therapy. Although these practices are not evidence based in the manner of modern Western medicine, they have been regarded by people indigenous to their respective communities as efficacious methods for the improvement of human well-being. Given their longevity as cultural practices and cheap and noninvasive nature, it is worthwhile for social scientific researchers to gain at least a general understanding of traditional modes of music and soundbased therapy that have been in use for hundreds, even thousands of years.

\section{Sound and Evidence-Based Healing}

\subsection{Music and healing}

Many cultures have used music for healing during ancient times, and music has often been used as a method of relaxation and reflection. However, even studies today show that music can be used as treatment for conditions such as heart disease, depression, and stroke [22]. In Music as Medicine, Simon Heather discusses several uses of music in healing and treatment, including 23 recent scientific studies that used music in the treatment of coronary heart disease. The results showed that of 1,500 participants, those who listened to music while being treated for their condition showed better health than those who received standard care [22]. Listening to music while being treated also had beneficial effects on blood pressure, heart and respiratory rates, anxiety, and pain [22]. 
It has also been shown that listening to music before or during surgery can reduce the level of anxiety and pain in adults and children [22]. One study showed that listening to music had the same level of relief as the use of sedatives in 207 patients before undergoing an operation [22]. "The researchers found no significant differences in anxiety, cortisol level, heart rate and blood pressure between those taking diazepam and those who listened to music in the run-up to surgery" [22]

A study in Helsinki, Finland, showed that listening to music for multiple hours every day can decrease recovery time in the early stages following a stroke [22]. "The results showed that the recovery of verbal memory and attention improved significantly more with the group of patients who listened to music compared with those patients who listened to audio books or did nothing at all" [22]. Participants who listened to music expressed that they also felt less depressed and confused than the group that did not listen to music. The difference between the two groups were still present 6 months later. This shows the importance of music in healing, indicating that music may have long-term effects on an individual's brain function.

One study showed the effectiveness of music in the treatment of depression. According to an article published in the Journal of Advanced Nursing, listening to music can reduce chronic pain by up to $21 \%$ and depression by $25 \%$ : "The people who took part in the music groups listened to music on a headset for an hour a day and everyone who took part, including the control group, kept a pain diary" [23].

Studies have also examined the positive effects of music on the treatment of patients with dementia. For example, Clive Holmes, a professor from Southampton University, studied the effects of live music on dementia patients and said: "Suddenly they came alive - some hadn't spoken for three years" [22]. A recent study explored the impact of live performances on patients with severe dementia. Researchers scored patients in terms of awareness and alertness while several live bands played. People that previously were too disabled to have a conversation danced and sang along with the music [22]. One burgeoning organization that is leading the way in creating innovative applications of music therapy for clients suffering from dementia is Music and Memory. Run by Dan Cohen, a social worker, Music and Memory "promotes the use of individualized music in nursing homes, assisted care facilities, and private homes" [24]. Cohen has noted that music therapy has a profound effect on the mood states, attention, and motivation of dementia clients while also having a distinctly positive impact in terms of reducing caregiver stress [24]. He estimated that the program works well in about $75 \%$ of cases, whereas the remaining $25 \%$ are cases in which clinicians have simply not yet found the correct personalized music for their clients [24].

The American Cancer Society has noted there is some evidence that music can help reduce pain and even relieve chemotherapy-induced nausea and vomiting [25]. Listening to music also relieves stress and encourages an overall sense of well-being. Although there is no scientific proof that music has the ability to cure diseases, studies have found that music therapy can lower heart rate, blood pressure, and breathing rate [25].

The Los Angeles Times published a March 2014 article revealing that Duma's Foundation for Neurosciences, Stroke, and Recovery used 30 pieces of music to test with 100 different patients, each listening to three pieces of music [26]. The results showed that music relieved physical tension in some patients with arthritis and helped patients with Alzheimer's regain memory [26].

As previously discussed, the Om mantra is highly revered in several cultures, with some referring to it as the sound from which all life is created. Although Om is often used in cultures that do not fully rely on the use of modern sciences for medical purposes, studies have shown the neurological effects of the Om mantra. Researchers at Sanjay Gandhi Postgraduate Institute of Medical Sciences in Lucknow performed a study published in February in Cognition and Emotion [27]. The study used MRI scanners to monitor the brain activity of $21 \mathrm{men}$. Three sound conditions were compared - the ancient chant of Om, a similar but nonmeaningful sound condition (the sound "Tom"), and another meaningful sound condition (the Hindi word "Aam"). "Listening to the Om sound activates areas of the bilateral cerebellum, left middle frontal gyrus and right precuneus. ... Listening to Om recruits neural systems implicated in emotional empathy". 


\section{International Journal of Innovative Research in Medical Sciences (IJIRMS) \\ ISSN (Online): 2455-8737, Vol. 01, Issue 06, August 2016 \\ Available online at $-\underline{w w w . i j i r m s . i n}$}

Listening to the Om sound can literally cause an individual to feel more aware of his feelings, making it easier to understand another person's feelings.

Music has proven to be effective in reducing stress, fighting diseases, and managing pain. Two markers of the hypothalamic-pituitary-adrenal axis, B-endorphin and cortisol, were found to decrease in a study that used music interventions [28]. The study also showed that music increased plasma cortisol, adrenocorticotropic hormone, prolactin, growth hormone, and norepinephrine levels. The effects of the music interventions were also dependent on the individual's music preference, personality type, current mood, and other personal differences. Individuals who listen to music after having surgical operations may also display decreasing serum cortisol levels [28]. Chanda and Levitin [28] stated: "Music initiates brainstem responses that, in turn, regulate heart rate, pulse, blood pressure, body temperature, skin conductance, and muscle tension, partly via noradrenergic neurons that regulate cholinergic and dopaminergic neurotransmission" (p. 186). Future studies would be improved by having participants describe how much they enjoyed the music and examining other factors such as distractions to generate a more valid set of findings regarding the effects of music interventions [28].

A 2012 study that used self-selected music with 50 of 100 participants during interventions in radiotherapy treatment for anxiety found that anxiety significantly reduced during initial radiotherapy treatment in both music and control groups; self-selected music did not significantly affect anxiety levels [29]. However, researchers found that participants with higher baseline anxiety would benefit more specifically from using self-selected music during treatment [29].

A previous study in 2011 in Taiwan featured 165 participants from 20 to 65 years of age. The study explored the effectiveness of headphone music versus broadcast music in decreasing anxiety. The results showed no significant difference, but both groups of participants showed a significant decrease in anxiety when listening to music [30].

One of the exciting areas of music therapy treatment is with patients with dementia and persistent aphasias. Popular research has shown that music can access areas of the conscious mind that were previously unreachable. One study of patients with aphasia from stroke explored the SIPARI method [31], which seeks to activate right hemispheric speech abilities in aphasia patients. This study measured response to treatment in patients suffering from global aphasia and Broca aphasia compared to a control group with no treatment. The study used the Aachen Aphasia Test following interventions that involved singing, intonation, speechadequate prosody, and rhythmic exercises [31]. Results of the study showed pronounced improvement in all areas of the experimental group versus the control group.

\section{Conclusion}

Wolfgang Mastnak has stated: "The variety of musictherapeutic results ensures that the effect of music on the psyche is based on a multifunctional process comprising physiological, emotional, and cognitive factors as well as on anthropological, cultural, and individual conditions" [32]. This means that the way music is processed in our minds is dependent on various factors. The loud, energetic, and bold tones and instrumentation of American rock and roll and hip-hop music may be palatable to one person, but another may not be accustomed to such sounds and find them unpleasant. Many people in today's world carry music files on small devices that are always at their fingertips. A person who is walking, driving, reflecting, celebrating, and exercising may listen to five or more types of music in one day, depending on her mood and environment. Once research unveils a clearer picture of the effects of music on our health and well-being, we will be able to make informed decisions about incorporating music and other healing sounds into spiritual, emotional, religious, and medical contexts.

\section{References}

[1] L. Garrison, "Kanye, Lamar Odom \& The Miraculous Healing Power of Music" May 10, 2015. [Online]. Available: http://djbooth.net/news/entry/2015-05-10-kanyelamar-odom-healing-music.

[2] Y. Kim, "The Integration of Eastern and Western Cultures in the Training of Music Therapists in Asia," in International Perspectives in Music Therapy Education and Training: Adapting to a Changing World, K. D. Goodman (ed.), Charles C Thomas Springfield, 2015. 
[3] H. M. Ridder, "An Overview of Therapeutic Initiatives when Working with People Suffering from Dementia," in Music Therapy and Neurological Rehabilitation: Performing Health, D. Aldridge (ed.), Jessica Kingsley, Philadelphia, 2005.

[4] S. K. Gilbertson, "Music Therapy in Neurorehabilitation after Traumatic Brain Injury: A Literature Review," in Music Therapy and Neurological Rehabilitation: Performing Health, D. Aldridge (ed.), Jessica Kingsley, Philadelphia, 2005.

[5] D. W. Moore, Rhythm Healing: PTSD Trauma and Beyond, Soul Dance, Olympia, 2013.

[6] H. M. Ridder, "Doctoral Education: A Model of Problem-Based Learning," in International Perspectives in Music Therapy Education and Training: Adapting to a Changing World, K. D. Goodman (ed.), Charles C Thomas, Springfield, 2015.

[7] D. K. Statnekov, Animated Earth: A Story of Peruvian Whistles and Transformation, North Atlantic Books, Berkeley, 2003.

[8] Metropolitan Chicago Healthcare Council, Guidelines for Healthcare Providers Interacting with American Indian (Native American: First Nation) Patients and Their Families, Committee on Clinical, Administrative, Professional, and Emergency Services, Chicago, 2004.

[9] Manataka American Indian Council, Native American Spirituality, Manataka American Indian Council, Hot Springs, 2006.

[10] F. Densmore, The American Indians and their Music (revised edition), Woman's Press, New York, 1936.

[11] E. B. Miller, C. F. Gross, "An Exploration of Physiological Responses to the Native American Flute," paper presented at ISQRMM 2013, Athens, GA, July 26, 2013.

[12] M. Muynck, Vibrational Healing Music, Linda Marie Walker, Santa Fe, 2009.

[13] T. Riggs, "Hinduism," in T. Riggs (ed.), Worldmark Encyclopedia of Religious Practices, Cengage Learning, Boston, 2006.

[14] T. Ashley-Farrand, Healing Mantras, Sounds True, Boulder, 1999.

[15] D. Frawley, Mantra Yoga and Primal Sound, Lotus Press, Twin Lakes, 2010.

[16] A. Mathur, S. H. Vijayakumar, B. Chakrabarti, N. C. Singh, "Emotional Responses to Hindustani
Raga Music: The Role of Musical Structure,' Frontiers in Psychology, 6(513), pp. 1-11, 2015.

[17] B. A. Wallace, S. L. Shapiro, "Mental Balance and Well-Being: Building Bridges between Buddhism and Western Psychology," American Psychology, 61(7), pp. 690-701, 2006.

[18] B. A. Wallace, "The Buddhist Tradition of Samatha: Methods for Refining and Examining Consciousness," Journal of Consciousness Studies, 6(2-3), pp. 175-187. 1999.

[19] Dalai Lama, H. C. Cutler, The Art of Happiness: A Handbook for Living, Riverhead Books, New York, 1998.

[20] S. Misra, I. Shastri, "Rumination of Music on Buddhism and Hinduism," Sport and Art, 2(3), pp. 33-40, 2014.

[21] L. M. K. Dona, "Community Music Therapy in Action: Healing through Pirit Chanting in Sri Lanka," Journal of Urban Culture Research, 1, pp. 148-156, 2010.

[22] S. Heather, "Music as Medicine," June 2010. [Online]. Available: http://www.simonheather.co.uk/pages/articles/mu sic as medicine.pdf.

[23] S. L. Siedlecki, "The Effect of Music on Power, Pain, Depression and Disability," Journal of Advanced Nursing, 54(5), pp. 553-562, 2005.

[24] S. Fisher, S. "Easing Dementia with Music," The Costco Connection, June, 2016.

[25] American Cancer Society, "Music Therapy," 2016. [Online]. Available: http://www.cancer.org/treatment/treatmentsandsi deeffects/complementaryandalternativemedicine/ mindbodyandspirit/music-therapy.

[26] J. Ogilvie, "Music as Suite Medicine," Los Angeles Times, p. E6, Mar. 22, 2014.

[27] U. Kumar, A. Guleria, C. L. Khetrapal, "NeuroCognitive Aspects of 'OM' Sound/Syllable Perception: A Functional Neuroimaging Study," Cognition and Emotion, 29(3), pp. 432-441, 2015.

[28] M. L. Chanda, D. J. Levitin, "The Neurochemistry of Music," Trends in Cognitive Sciences, 17(4), pp. 179-193, 2013.

[29] C. O'Callaghan, M. Sproston, K, Wilkinson, D. Willis, A. Milner, D. Grocke, G. Wheeler, "Effect of Self-Selected Music on Adults' Anxiety and Subjective Experiences during Initial Radiation Treatment: A Randomised Controlled Trial and Qualitative Research," 
Journal of Medical Imaging and Radiation Oncology, 56(4), pp. 473-477, 2012.

[30] K.-C. Lee, Y.-H. Chao, J.-J. Yiin, P.-Y. Chiang, Y.-F. Chao, "Effectiveness of Different MusicPlaying Devices for Reducing Preoperative Anxiety: A Clinical Control Study," International Journal of Nursing Studies, 48, pp. 1180-1187, 2011.

[31] M. Jungblut, "Music Therapy for People with Chronic Aphasia: A Controlled Study," in Music Therapy and Neurological Rehabilitation: Performing Health, D. Aldridge (ed.), Jessica Kingsley, Philadelphia, 2005.

[32] W. Mastnak, "Non-Western Practices of Healing Music and Applications for Modern Psychotherapy," International Review of the Aesthetics and Sociology of Music, 24(1), pp. 7784, 1993.

\section{Special Acknowledgement:}

Eric Lindberg, editor - writer, the Hamovitch Center for Science in the Human Services, University of Southern California, School of Social Work

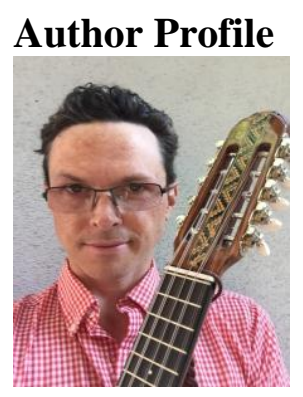

Nils de Mol van Otterloo received BA in Music from Bates College in 1998, BM from Berklee College of Music in 2001, and MSW from University of Southern California School of Social Work in 2015. He is doing research in dementia care and music at Amrita University as a Fulbright Scholar from 2016 through 2017.

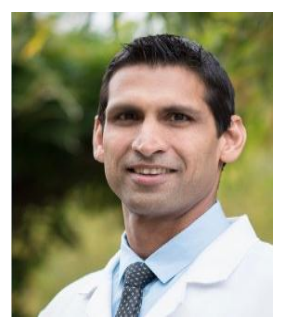

Nimit Sudan, M.D., joined City of Hope in 2012 from the Yolanda G. Barco Oncology Institute at the Meadville Medical Center in Meadville, Pennsylvania where he was the Director of Medical Oncology. Concurrently, he held the post of adjunct clinical assistant professor at Lake Erie College of Osteopathic Medicine. Dr. Sudan is board certified in Internal Medicine, Hematology and Oncology, and is also certified in Acupuncture. He has published several peer reviewed manuscripts and is a recipient of the Cancer Liaison Physician Outstanding Performance Award presented by the Commission on Cancer in 2010. He is a member of several professional organizations, including the American Society of Hematology and the American Society of Clinical Oncology.

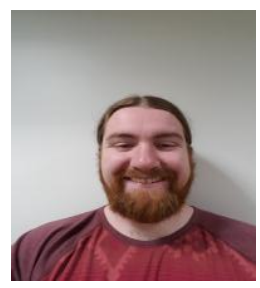

Matthew Lyons is a doctoral student in Public Health at Georgia State University. His research interests include the social determinants of mental health, substance abuse, homelessness, intervention development, and research dissemination. He holds a Master of Social Work degree from the University of Southern California and a Master of Theological Studies from Harvard Divinity School.

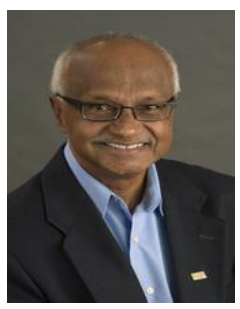

Murali Nair, PhD is a Clinical Professor of Social Work and Affiliate of the Institute of Integrative Health at the University of Southern California. Prior to that he was the founding CoDirector of the Center for Healing Across Cultures at the Cleveland State University. He received the Doctor of Social Work (DSW) and PhD from Columbia University and a Master of Computer Science (MS) from the New York Institute of Technology. At present, Professor Nair is involved in cross national field studies on understanding the "Life style factors of Healthy Centenarians". Several of his articles and mini documentaries can be found on his website: www.muralinair.com.

\section{Additional Resources}

http://www.musictherapy.org/ - The website of the American Music Therapy Association.

https://www.berklee.edu/music-therapy - Page detailing the music therapy major available at Berklee College of Music.

http://www.csun.edu/mike-curb-arts-mediacommunication/music/music-therapy-clinic-information - Website for the CSUN music therapy clinic, which provides individual music therapy services for people with disabilities.

http://gsmusictherapy.com/ - Website for an organization that provides music therapy services for older adults, children, and people with disabilities in California.

http://www.cnn.com/videos/tv/2015/01/08/musictherapy.cnn - A CNN profile on music therapy.

http://www.themusictherapycenter.com/ - Website of the Music Therapy Center of California, which is a music therapy organization in San Diego specializing in serving youth with special needs. 